\title{
Síntomas depresivos y obesidad en adolescentes. Una aplicación desde la Minería de Datos $^{9}$
}

\author{
Norma Cecilia Serrano Díaz \\ Médica y genetista. Magister en bioética \\ Fundación Cardiovascular de Colombia \\ Correo electrónico: normaserrano@fcv.org \\ David Andrés Castro Ruiz \\ Magister en Gestión, Aplicación \\ y Desarrollo de Software \\ Fundación Cardiovascular de Colombia \\ Correo electrónico: david.castroruiz@gmail.com
}

\author{
Paula Fernanda Pérez Rivero \\ Magíster en Ciencias Básicas Biomédicas \\ Especialista en Psicología Clínica \\ Universidad Pontificia Bolivariana Seccional Bucaramanga \\ Correo electrónico: paula.perez@upb.edu.co
}

\author{
Diana Paola Suárez Suárez \\ Enfermera \\ Fundación para la Excelencia de la \\ Medicina Clínica en Colombia \\ Fundación Cardiovascular de Colombia \\ Correo electrónico: dianasuarez@fcv.org
}

Doris Cristina Quintero-Lesmes

PhD. demografía

Fundación Cardiovascular de Colombia Correo electrónico: dorisquintero@fcv.org
Recibido: 08/06/2020

Evaluado: $11 / 08 / 2020$

Aceptado: 26/08/2020

\section{Resumen}

Objetivo: describir mediante la aplicación de minería de datos quién desarrolla obesidad y síntomas depresivos (SD) en la población adolescente de la ciudad de Bucaramanga. Métodos: a través de un estudio descriptivo y transversal anidado en una cohorte poblacional. Se evaluaron 432 adolescentes. Se captó información de variables sociodemográficas, SD y medidas antropométricas. Se llevaron a cabo análisis estadísticos para variables categóricas y continuas, así como un análisis de minería de datos. Resultados: el $26.7 \%$ de los adolescentes presentó exceso de peso. Se observó que más de la mitad de la muestra presentó SD. El análisis de minería de datos permitió identificar seis grupos de participantes de acuerdo con sus características con relación al peso y a los SD. Conclusiones: la relación entre SD y obesidad se estableció en el grupo de mujeres adolescentes, observándose que aquellas con un peso mayor a 2 desviaciones estándar presentaban todos los SD.

Palabras clave Obesidad, Sobrepeso, Depresión, Adolescente, Estilo de vida, Minería de datos.

9 Para citar este artículo: Serrano, N., Castro, D., Pérez, P., Suárez, D.P. y Quintero-Lesmes, D.C. (2022). Síntomas depresivos y obesidad en adolescentes. Una aplicación desde la Minería de Datos. Informes Psicológicos, 22(1), pp. 151-166 http://dx.doi.org/10.18566/infpsic.v22n1a09 


\section{Depressive symptoms and obesity in adolescents. An application from Data Mining}

Abstract

Objective: to describe who develops obesity and depressive symptoms (DS) in the adolescent population of the city of Bucaramanga by means of the data mining application. Methods: a descriptive and cross-sectional study nested in a population cohort was applied. 432 adolescents were evaluated. Information on sociodemographic variables, DS and anthropometric measures was collected. Statistical analyzes were carried out for categorical and continuous variables, as well as a data mining analysis. Results: $26.7 \%$ of the adolescents were overweight. It was observed that more than half of the sample presented DS. The data mining analysis allowed the identification of six groups of participants according to their characteristics in relation to weight and DS. Conclusions: the relationship between DS and obesity was established in the group of adolescent women, observing that those with a weight greater than 2 standard deviations presented all DS.

Keywords

Obesity, Overweight, Depression, Adolescent, Lifestyle, Data mining.

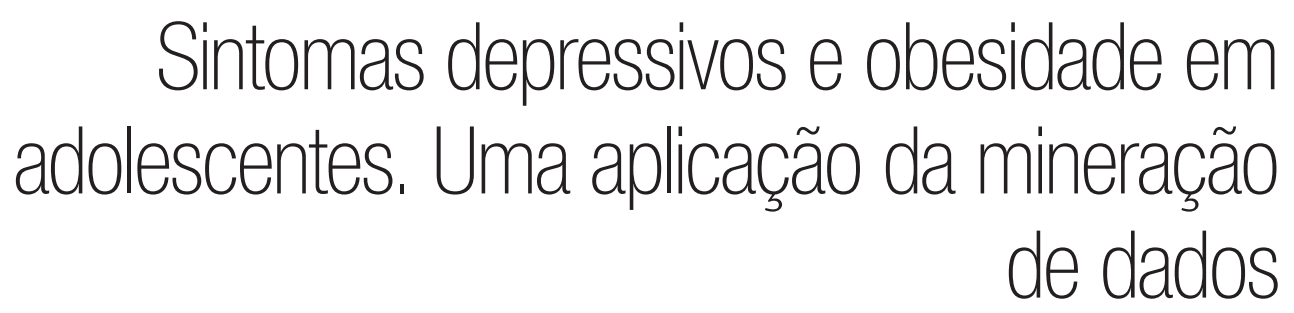

Resumo

Objetivo: descrever por meio da aplicação de mineração de dados quem desenvolve obesidade e sintomas depressivos (SD) na população adolescente da cidade de Bucaramanga. Métodos: por meio de um estudo descritivo e transversal aninhado em uma coorte populacional. Foram avaliados 432 adolescentes. Foram coletadas informações sobre variáveis sociodemográficas, DP e medidas antropométricas. As análises estatísticas foram realizadas para variáveis categóricas e contínuas, bem como uma análise de mineração de dados. Resultados: $26.7 \%$ dos adolescentes estavam com sobrepeso. Observou-se que mais da metade da amostra apresentou SD. A análise de mineração de dados permitiu a identificação de seis grupos de participantes de acordo com suas características em relação ao peso e SD. Conclusões: a relação entre SD e obesidade foi estabelecida no grupo de mulheres adolescentes, observando-se que aquelas com peso maior que 2 desvios-padrão apresentaram todos SD.

Palavras chave Obesidade, Sobrepeso, Depressão, Adolescente, Estilo de vida, Mineração de dados. 


\section{ntroducción}

En las últimas dos décadas ha aumentado el uso de técnicas de minería de datos en varias disciplinas (Dipnall et al., 2016). La minería de datos incorpora un camino hacia el descubrimiento de conocimiento y es un proceso importante para descubrir patrones en los datos mediante la exploración y el modelado de datos. En disciplinas de la salud, estos patrones pueden ser utilizados para establecer diagnósticos y pronósticos que mejoren la calidad de los tratamientos, reduciendo significativamente los costos tanto en las instituciones de salud como de la población general (Milovic \& Milovic, 2012). La minería de datos incorpora un aprendizaje automático mediante algoritmos para extraer e identificar información útil; estas técnicas de análisis de datos se han utilizado en la investigación médica, principalmente en neurociencia y biomedicina (Pirooznia et al., 2012). Más recientemente, la psiquiatría ha comenzado a utilizar los beneficios de estas técnicas para obtener una mejor comprensión de la enfermedad mental, especialmente de la depresión (Ni et al., 2014).

La depresión es un trastorno mental frecuente cuya sintomatología incluye: anhedonia, tristeza, pérdida de interés, sentimientos de culpa, pobre autoestima, trastornos del sueño y de la alimentación, cansancio, fatiga y problemas cognitivos (fallas en la atención y memoria principalmente) (Organización Mundial de la Salud, [OMS] 2017). Los trastornos depresivos en general son crónicos y recurrentes y afectan dramáticamente la vida activa de la población, considerándose como una de las principales causas de discapacidad y pérdida de años productivos. De acuerdo con la OMS (2017) hay en el mundo 350 millones de personas diagnosticadas con algún tipo de depresión y debido a sus implicaciones se ha declarado un problema de salud pública.

En Colombia, se han realizado investigaciones descriptivas que dan cuenta de la presencia de la depresión en la población. En el año 2015, el Sistema Integral de Información de la Protección Social (SISPRO) reportó que el trastorno depresivo fue diagnosticado a 36,584 personas. En relación con el sexo, se observó que el diagnóstico, tanto de depresión moderada como severa, se presentó más en mujeres $(70.4 \%$ de los casos) que en hombres (29.6\%). En cuanto a los grupos etarios, se observó que los casos atendidos crecieron a medida que aumentaba la edad hasta alcanzar el punto máximo en el grupo de los 50 a 54 años. Sin embargo, para la depresión psicótica los grupos con más frecuencia fueron de los 20 a los 24 años y de 75 años en adelante. En el grupo de niños y adolescentes se observó un mayor diagnóstico en las edades de 10 a 14 años (1500 casos) siendo evidente una tendencia al aumento desde el 2009, año en que se registraron solo 991 casos (Ministerio de Salud, 2017). En el análisis por departamentos, se halló que en Santander en el 2009 se había diagnosticado con depresión a 991 personas mientras que en el 2015 a 1754. Los departamentos con mayor prevalencia fueron Antioquia, Bogotá y Valle del Cauca (Ministerio de Salud, 2017).

Por otro lado, uno de los problemas de salud pública mundial más grave del 
siglo XXI es la obesidad, definida como una acumulación anormal o excesiva de grasa provocada por un desequilibrio crónico entre la ingesta y el consumo de energía, convirtiéndose en un riesgo para la salud cardiovascular y metabólica de la población general (OMS, 2002; 2010). En Colombia, 1 de cada 6 niños y adolescentes presenta sobrepeso u obesidad (Ministerio de Salud, 2015), y en Santander, la prevalencia de exceso de peso en esta población aumentó $2.4 \%$ del año 2010 (15.5\%) al año 2015 (17.9\%), según la Encuesta Nacional de Situación Nutricional (Ministerio de Salud, 2015).

En los últimos años, la obesidad se contempla no sólo como un factor predisponente para las complicaciones fisiológicas, sino que además parece estar relacionada con trastornos psicopatológicos, lo que explicaría el aumento de la prevalencia de personas obesas con padecimientos psiquiátricos y psicológicos, en comparación con la población general. La evidencia científica ha mostrado que existe una asociación entre obesidad y depresión (Pereira-Miranda, Costa, Queiroz, Pereira-Santos \& Santana, 2017).

En adolescentes, la relación entre obesidad y depresión parece ser bidireccional, tal y como señalan Luppino et al. (2010) quienes llevaron a cabo un metaanálisis de 15 estudios longitudinales que evaluaban la relación entre ambas variables. Los hallazgos mostraron que los adolescentes con trastornos depresivos y con síntomas depresivos tenían más riesgo de desarrollar obesidad, siendo mayor en aquellos con diagnóstico clínico. Igualmente, aquellos individuos con obesidad en la adolescencia tenían más riesgo de presentar algún trastorno depresivo durante la adultez.
En esta misma línea, Quek, Tam, Zhang y Ho (2017) realizaron un metaanálisis con 18 investigaciones que estudiaban la asociación entre obesidad y depresión en la infancia y la adolescencia. Sus resultados mostraron que las niñas y mujeres adolescentes con obesidad tenían más riesgo de presentar síntomas más severos de depresión que los hombres con obesidad y que las mujeres y hombres con peso normal.

Los reportes de estos metaanálisis son consistentes con lo que han mostrado varias investigaciones, en las que las personas con obesidad presentan más riesgo de sufrir depresión (Cabas, González \& Mendoza, 2018), en especial las mujeres (Martinson \& Vasunilashorn, 2016). También con estudios que señalan que la depresión en la adolescencia aumenta el riesgo de padecer obesidad (Lasserre et al., 2014; Milano et al., 2020). En este sentido, existe evidencia que apoya la idea de la relación bidireccional entre obesidad y depresión en este grupo etario.

El interés por estudiar esta relación ha surgido quizás debido a las implicaciones que tiene para la salud emocional de los individuos aspectos relacionados con su imagen corporal y la funcionalidad (Stice \& Desajardins, 2018) y también porque la depresión es un trastorno que afecta las habilidades de autocuidado de las personas y dentro de sus síntomas incluye desórdenes alimenticios (Ranta et al., 2017). No obstante, a pesar del cúmulo de resultados obtenidos hasta el momento, no se ha podido determinar con certeza la naturaleza de esta asociación debido a la multiplicidad de métodos aplicados y la falta de control de sesgos de algunas investigaciones. 
El desarrollo de dos situaciones patológicas (síntomas depresivos versus obesidad) de impacto en la salud infantil y el interés de analizar los datos desde otras técnicas de la bioinformática, definieron el objetivo de este trabajo que fue describir mediante una aplicación de minería de datos quién desarrolla obesidad y depresión en la adolescencia en la ciudad de Bucaramanga, Colombia.

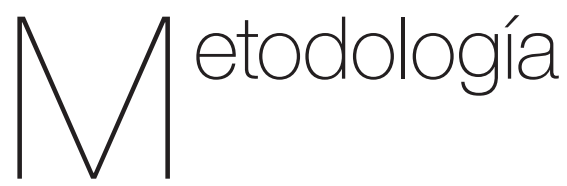

El presente estudio fue descriptivo y transversal, anidado en una cohorte poblacional. Del total de la población de escolares $(1,282)$ pertenecientes a una cohorte población (Villa-Roel et al., 2009) de la ciudad de Bucaramanga, se evaluaron 494 participantes en su edad adolescente. Se excluyeron 62 participantes por tener información incompleta en sus formularios.

Los investigadores del estudio entrenaron a profesionales de la salud para que realizaran la fase de recolección de datos, esto con el fin de garantizar el uso de técnicas estandarizadas definidas previamente. De modo que se garantizara la calidad de los datos, a través de Excel (Microsoft Corp., Redmond, Wash), se llevó a cabo digitación por duplicado. También se compararon las bases de datos con el software Epi-Info 2000. En caso de observarse discrepancias en los resultados de ambos programas, se procedía a corregir los datos comparándolos con los formatos originales de recolección.

\section{Evaluación de los síntomas depresivos}

Se definió por una serie de preguntas que contestaron los participantes (autoreporte), y que evaluaron la frecuencia con la que el individuo entrevistado realizaba alguna acción específica durante 12 meses anteriores a la entrevista como: cansancio para realizar labores diarias o problemas para dormir, sentimientos como tristeza, infelicidad, preocupación y nerviosismo, así como la percepción o relación que tenía el individuo con los demás. El cuestionario fue elaborado teniendo en cuenta los criterios consignados en la Clasificación Internacional de Enfermedades, versión 10 (OMS, 1994).

\section{Evaluación del estado nutricional}

Los datos antropométricos fueron recolectados de acuerdo con los lineamientos de Lohman Roche y Martorell (1988). Para el peso, obtenido en kilogramos, se utilizó una báscula digital (Seca 813), la cual cuenta con una sensibilidad de .1 kilogramos. La estatura, medida en centímetros, se registró con un estadiómetro portátil (Seca 206), cuya sensibilidad fue de .1 centímetros. El perímetro de cintura y cadera, se tomaron con una cinta métrica (Seca 201), con una sensibilidad de .1 centímetros. La toma de los pliegues cutáneos bicipital, tricipital, supra ilíaco y subescapular fue llevada a cabo con un plicómetro (Slimguide) el cual tiene una precisión de .5 milímetros. El estado nutricional se determinó con los siguientes parámetros: 
a. Índice de masa corporal (IMC) ajustado para edad y sexo: se dividió el peso en kilogramos sobre la estatura en metros cuadrados; para su clasificación se utilizaron los puntos de corte de la OMS-2007 (De Onis et al., 2007) siendo bajo: percentil <3, eutrófico: percentil 3 a 85, sobrepeso: percentil > 85 a 97 y obeso: percentil $>97$.

b. I Índice Cintura / Cadera (ICC): se estimó dividiendo el perímetro de la cintura sobre el perímetro de la cadera en centímetros.

c. Índice Cintura / Talla: se calculó como el cociente entre el perímetro de la cintura (centímetros) sobre la estatura (cm).

d. Porcentaje de grasa corporal total: se usó la ecuación de Slaughter (Curilem-Gatica et al., 2016) con los pliegues tricipital y subescapular (material suplementario).

\section{Variables sociodemográficas}

Se recolectaron las siguientes: a. Variables sociodemográficas: sexo, edad en años y nivel socio económico. b. Antecedentes: duración de la lactancia materna (meses), antecedentes de tabaquismo y consumo de alcohol (para ambas variables se preguntó si alguna vez había fumado o bebido), tiempo de exposición a pantallas (horas/semana) y actividad física (horas/semana).

\section{Análisis Estadístico}

Las variables categóricas se presentaron como proporciones; para establecer las diferencias entre éstas se utilizó Chi cuadrado o prueba exacta de Fisher, según correspondía. Para las variables continúas, considerando su distribución, se recurrió a las medianas como medida de tendencia central y al rango intercuartílico como medida de variabilidad. Las diferencias por sexo fueron estimadas con $U$ de Mann Whitney. Los análisis se realizaron con el programa estadístico Stata, versión 14.0 (College Station, TX: Stata Corporation). Se reportaron los datos descriptivos e inferenciales de aquellas variables que resultaron significativas en los análisis.

Por otra parte, se llevó a cabo un análisis con técnicas de minería de datos o datamining. La minería de datos es un campo de la estadística y las ciencias de la computación que busca patrones ocultos en grandes volúmenes de datos que pueden aportar información valiosa para la toma de decisiones. Utiliza el análisis matemático para deducir los patrones y tendencias que existen en los datos; para este caso, se realizó un proceso de extracción del conocimiento o KDD (Knowledge Discovery in Databases), que se refiere al proceso no-trivial de descubrir conocimiento e información potencialmente útil dentro de los datos contenidos en algún repositorio de información. KDD es una metodología propuesta por Fayyad, Piatetsky-Shapiro y Smyth (1996) en cinco fases: selección de datos, pre-procesamiento, transformación, minería de datos y evaluación e implantación.

a) Selección de datos: se determinaron las fuentes de datos y el tipo de información a utilizar. Para este análisis 
se seleccionó una población de 494 participantes provenientes del estudio SIMBA y se escogieron variables de tipo: sociodemográficas, antropométricas y síntomas de depresión.

b) Pre-procesamiento: consistió en la preparación y limpieza de los datos escogidos. Se eliminaron participantes con información insuficiente (62 participantes). Se recodificaron las categorías de valores numéricos a valores alfa-numéricos (ej. Sexo 0 = mujer y 1 = hombre; síntomas depresivos 0 $=\mathrm{No}, 1=\mathrm{Si}$ )

c) Transformación: se llevó a cabo el tratamiento preliminar de los datos, transformación y generación de nuevas variables a partir de las ya existentes con una estructura de datos apropiada. En esta base de datos no se realizaron procesos posteriores a los ya mencionados en el paso anterior.

d) Minería de datos: fueron aplicados métodos inteligentes con el objetivo de extraer patrones previamente desconocidos, válidos, nuevos, útiles y comprensibles, que están ocultos en los datos (Two Crows Corporation, 2005). Para el set de datos escogido se procedió a aplicar un algoritmo de agrupamiento llamado KMeans, utilizando el software Weka (Waikato Environment for Knowledge Analysis) versión 3.8 (Weka Company, s.f.).

e) Interpretación y evaluación: se identificaron los patrones obtenidos y se realizó una evaluación de los resultados, de acuerdo con las medianas con sus respectivos rangos intercuartílicos y frecuencias relativas en cada una de las agrupaciones (clúster).

\section{Consideraciones éticas}

El estudio que soportó las evaluaciones de los participantes en la edad adolescente fue revisado y aprobado por el Comité de Ética en Investigaciones de la Fundación Cardiovascular de Colombia en acta No. 396 de 9 abril de 2016. El consentimiento fue obtenido por parte de los padres o representantes legales de los participantes. Además, todos los participantes menores de edad dieron su asentimiento verbal y por escrito. Los adolescentes mayores de edad dieron su consentimiento por escrito.

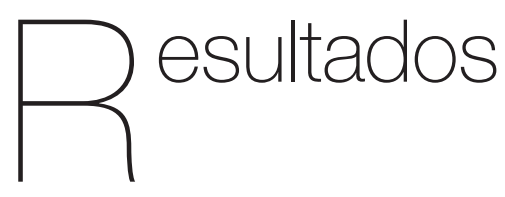

\section{Características de la muestra}

La muestra incluyó 432 adolescentes, residentes de la ciudad de Bucaramanga, participantes de la cohorte original (20062007). La mediana de edad fue de 16.5 años (rango Intercuartílico, RIC: 2.7 años) sin diferencias por sexo. Se encontró que el $55.5 \%$ de los adolescentes provenía de un nivel socio económico bajo. No se hallaron diferencias al comparar por sexo (ver Tabla 1).

\section{Antecedentes}

Haber fumado (38\%) fue menor comparado con el consumo de alcohol (72.1\%), siendo éste mayor en hombres (44.1\%) que en mujeres (34.4\%). En relación con 
el tiempo de exposición a pantallas (teléfonos móviles, tabletas, televisor y otros dispositivos) no se observaron diferencias significativas entre hombres y mujeres (ver Tabla 1).

\section{Medidas antropométricas}

Se encontraron mayores valores de taIla, índice cintura- cadera y presión arterial sistólica en hombres que en mujeres $(p<$ .001). De forma semejante, la prevalencia de mayor peso fue superior en mujeres que en hombres $(p<.001)$.

Adicionalmente, el $26.7 \%$ de los adolescentes tuvo un exceso de peso, distribuido en un $12.5 \%$ con sobrepeso, siendo mayor en mujeres (13.9\%) comparado con hombres (8.3\%). Por otra parte, el 16.5\% presentó obesidad, sin observarse diferencias significativas entre mujeres y hombres (14.5\% vs. 17.1\%). No se hallaron diferencias significativas en los valores de presión arterial diastólica según el sexo (ver Tabla 1).

\section{Síntomas de la depresión}

En cuanto a la depresión se encontró que al menos el $50 \%$ de los participantes presenta ocho indicadores de este trastorno. El síntoma más frecuente fue el de sentirse cansado todo el tiempo $(n=332$, $76.8 \%$ ) seguido por sentir que las demás personas actúan mejor que uno mismo ( $n=279,64.5 \%)$, sentirse nervioso ( $n=$ $277,64.1 \%$ ), cambios en el apetito ( $\mathrm{n}=$ 237, 54.8\%), tener problemas para dormir ( $n=226,52.3 \%$ ), sentirse infeliz ( $n=226$, $52.3 \%$ y percibir que los demás no le tratan con respeto ( $n=219,50.6 \%$ ).

Tabla 1.

Características de los participantes en el estudio, según el sexo.

\begin{tabular}{|c|c|c|c|c|}
\hline Características & $\begin{array}{c}\text { Total } \\
n=432\end{array}$ & $\begin{array}{l}\text { Mujeres } \\
\mathrm{n}=226\end{array}$ & $\begin{array}{c}\text { Hombres } \\
n=206\end{array}$ & Valor $\mathrm{p}$ \\
\hline \multicolumn{5}{|l|}{ Variables socio-demográficas } \\
\hline Edad en años, (mediana [RIC]) & $16.5(2.7)$ & $16.5(2.5)$ & $16.6(2.9)$ & $.350 ¥$ \\
\hline \multicolumn{5}{|l|}{ Nivel Socio económico, n (\%) } \\
\hline Bajo & $240(55.5)$ & $129(57.0)$ & $111(53.8)$ & $.714 \dagger$ \\
\hline Medio & $187(43.2)$ & $94(41.5)$ & $93(45.1)$ & \\
\hline Alto & $5(1.1)$ & $3(1.3)$ & $2(.97)$ & \\
\hline \multicolumn{5}{|l|}{ Antecedentes, n (\%) } \\
\hline Duración de la lactancia materna (meses) & $12(18.0)$ & $12(18)$ & $12(18)$ & $.371 ¥$ \\
\hline Haber fumado al menos una vez en la vida & $188(38)$ & $35(13.9)$ & $39(16.8)$ & $.351 ¥$ \\
\hline Haber consumido alcohol al menos una vez en la vida & $188(72.1)$ & $86(34.4)$ & $102(44.1)$ & $.028 ¥$ \\
\hline \multicolumn{5}{|l|}{ Tiempo de exposición a pantallas (horas/semana) } \\
\hline Menos de 4 horas & $282(65.2)$ & $149(65.9)$ & $133(64.5)$ & $.766 ¥$ \\
\hline 4 horas o más horas & $150(34.7)$ & $77(34.0)$ & $73(35.4)$ & \\
\hline \multirow[t]{2}{*}{ Actividad física moderada e intensa (horas/ semana) } & $2.25(1.1)$ & $2.31(1.12)$ & $2.25(1.12)$ & $.969 ¥$ \\
\hline & & & & Contin \\
\hline
\end{tabular}


Continuación

\begin{tabular}{|c|c|c|c|c|}
\hline Características & $\begin{array}{c}\text { Total } \\
n=432\end{array}$ & $\begin{array}{l}\text { Mujeres } \\
\mathrm{n}=226\end{array}$ & $\begin{array}{c}\text { Hombres } \\
n=206\end{array}$ & Valor $p$ \\
\hline \multicolumn{5}{|l|}{ Variables antropométricas. (mediana [RIC]) } \\
\hline Talla (cm) & $163(13.0)$ & $158.5(7.3)$ & $169.9(8.5)$ & $<.001 ¥$ \\
\hline Peso (kg) & $57.0(17.9)$ & $60.3(16.8)$ & $53.1(14.4)$ & $<.001 ¥$ \\
\hline Índice de Masa Corporal (kg/m²) & $21.1(5.1)$ & $21.1(5.0)$ & $21.1(4.9)$ & $.245 ¥$ \\
\hline \multicolumn{5}{|l|}{ Estado nutricional según IMC/E. n (\%) } \\
\hline Bajo peso (<p3) & $58(13.1)$ & $35(15.4)$ & $23(11.0)$ & $.003 \dagger$ \\
\hline Peso normal (p3 - p85) & $263(60.8)$ & $133(58.5)$ & $130(62.9)$ & \\
\hline Sobrepeso (> p85 a p97)) & $44(10.1)$ & $21(8.7)$ & $23(11.4)$ & \\
\hline Obesidad ( $\geq$ p97) & $67(15.8)$ & $37(17.1)$ & $30(14.5)$ & \\
\hline Circunferencia de cintura (cm) & $74.5(11.9)$ & $73.8(11.9)$ & $75.3(13.0)$ & $.035 ¥$ \\
\hline Circunferencia de la cadera (cm) & $91.7(12.7)$ & $92.7(11.8)$ & $90.7(12.9)$ & $.004 ¥$ \\
\hline Índice cintura cadera & $0.82(.11)$ & $0.80(.12)$ & $0.84(.09)$ & $<.001 ¥$ \\
\hline Índice cintura talla & $0.45(.04)$ & $0.46(.07)$ & $0.44(.07)$ & $.0008 ¥$ \\
\hline Porcentaje de Grasa Corporal & $25.3(10.2)$ & $27.8(6.0)$ & $19.7(11.3)$ & $<.001 ¥$ \\
\hline \multicolumn{5}{|l|}{ Variables síntomas depresivos, $\mathrm{n}(\%)$} \\
\hline \multicolumn{5}{|c|}{ Estar muy cansado para hacer cualquier cosa (últimos 12 meses) } \\
\hline No & $100(23.1)$ & $38(16.8)$ & $62(30.1)$ & $.001 \ddagger$ \\
\hline $\mathrm{Si}$ & $332(76.8)$ & $188(83.1)$ & $144(69.9)$ & \\
\hline \multicolumn{5}{|c|}{ Presentar problemas para dormir (últimos 12 meses) } \\
\hline No & $206(47.6)$ & $100(44.2)$ & $106(51.4)$ & $.134 \ddagger$ \\
\hline $\mathrm{Si}$ & $226(52.3)$ & $126(55.7)$ & $100(48.5)$ & \\
\hline \multicolumn{5}{|c|}{ Sentirse triste, infeliz, deprimido (últimos 12 meses) } \\
\hline No & $206(47.6)$ & $82(36.2)$ & $124(60.1)$ & $<.001 \neq$ \\
\hline $\mathrm{Si}$ & $226(52.3)$ & $144(63.7)$ & $82(39.8)$ & \\
\hline \multicolumn{5}{|l|}{ Sentirse nervioso (últimos 12 meses) } \\
\hline No & $155(35.8)$ & $69(30.5)$ & $86(41.7)$ & $.015 \ddagger$ \\
\hline $\mathrm{Si}$ & $277(64.1)$ & $157(69.4)$ & $120(58.2)$ & \\
\hline \multicolumn{5}{|l|}{ Sentirse inútil (últimos 12 meses) } \\
\hline No & $287(66.4)$ & $138(61.0)$ & $149(72.3)$ & $.013 \ddagger$ \\
\hline $\mathrm{Si}$ & $145(33.5)$ & $88(38.9)$ & $57(27.6)$ & \\
\hline \multicolumn{5}{|l|}{ Cambios en el apetito (últimos 12 meses) } \\
\hline No & $195(45.1)$ & $77(34.0)$ & $118(57.2)$ & $<.001 \neq$ \\
\hline $\mathrm{Si}$ & $237(54.8)$ & $149(65.9)$ & $88(42.7)$ & \\
\hline \multicolumn{5}{|c|}{ Ha sentido que lo tratan con menos respeto que a otras personas. } \\
\hline No & $213(49.3)$ & $103(45.5)$ & $110(53.4)$ & $.104 \ddagger$ \\
\hline $\mathrm{Si}$ & $219(50.6)$ & $123(54.4)$ & $96(46.6)$ & \\
\hline \multicolumn{5}{|c|}{ Sentir que las personas actúan mejor que uno mismo (últimos 12 meses) } \\
\hline No & $153(35.4)$ & $80(35.4)$ & $73(35.4)$ & $.993 \ddagger$ \\
\hline $\mathrm{Si}$ & $279(64.5)$ & $146(64.6)$ & $133(64.5)$ & \\
\hline
\end{tabular}




\begin{tabular}{lcccc}
\hline \multicolumn{1}{c}{ Características } & $\begin{array}{c}\text { Total } \\
\mathbf{n}=\mathbf{4 3 2}\end{array}$ & $\begin{array}{c}\text { Mujeres } \\
\mathbf{n}=\mathbf{2 2 6}\end{array}$ & $\begin{array}{c}\text { Hombres } \\
\mathbf{n}=\mathbf{2 0 6}\end{array}$ & Valor p \\
\hline Le han puesto apodos o lo han insultado & & & & \\
No & $305(70.6)$ & $173(76.5)$ & $132(64.0)$ & $.004 \ddagger$ \\
$\mathrm{Si}$ & $127(29.4)$ & $53(23.4)$ & $74(35.9)$ & \\
Las personas se burlan del aspecto físico & & & & $.505 \ddagger$ \\
No & $308(71.3)$ & $158(69.9)$ & $150(72.8)$ & \\
Si & $124(28.7)$ & $68(30.0)$ & $56(27.1)$ & \\
Las personas se burlan del peso & & & & \\
No & $326(75.6)$ & $163(72.4)$ & $163(79.1)$ \\
Si & $105(24.3)$ & $62(27.5)$ & $43(20.8)$ \\
\hline
\end{tabular}

Nota. IMC/E: Índice de Masa Corporal para la Edad; *Determinado por Cuestionario Internacional de Actividad Física (IPAQ); RIC: Rango Intercuartílico. †: Valor p determinado mediante prueba exacta de Fisher. $\ddagger$ : Valor $p$ determinado mediante prueba Chi². $¥:$ Valor $p$ determinado mediante prueba $\mathrm{U}$ de Mann Whitney.

Adicionalmente, se halló que existen diferencias significativas entre los grupos de hombres y mujeres para los síntomas de: estar muy cansado $(p=.001)$, sentirse triste, infeliz o deprimido $(p<.001)$, sentirse nervioso ( $p=.015)$, sentirse inútil ( $p$ $=.013$ ), haber experimentado cambios en el apetito $(p<.001)$ y haber sido insultado o haber recibido apodos ofensivos ( $p=$ .004). En todos los casos se observó una mayor frecuencia de los síntomas en el grupo de las mujeres.

\section{Minería de Datos}

Al aplicar el algoritmo de agrupamiento (clustering), se realizaron varios análisis con diferente número de clúster, encontrando una distribución similar desde el clúster 6 en adelante donde se observó que los grupos quedaban repartidos con un número de instancias muy cercano, distribuyéndose porcentualmente entre el $12 \%$ y el $20 \%$. El análisis final de los resultados se realizó de acuerdo con la cantidad de clúster mencionados (6), por ser el que mejor distribuía el set datos (ver Tabla 2).
La edad de los participantes estuvo entre los 16 y 17 años, cuatro clústeres fueron para mujeres y dos para hombres. A continuación, se mencionan algunas características de cada grupo:

Clúster 0: Mujeres, con peso de 51.1 kilogramos y 163.0 metros de estatura, con 8 síntomas de depresión.

Clúster 1: Hombres, con peso de 68.8 kilogramos y 169.4 metros de estatura, con 0 síntomas de depresión.

Clúster 2: Mujeres, con peso de 59.8 kilogramos y 159.9 metros de estatura, con 7 síntomas de depresión.

Clúster 3: Mujeres, con peso de 72.2 kilogramos y 163.6 metros de estatura, con todos los síntomas de depresión, 11 en total.

Clúster 4: Hombres, con peso de 54.0 kilogramos y 167.7 metros de estatura, con 2 síntomas de depresión.

Clúster 5: Mujeres, con peso de 51.6 kilogramos y 158.0 metros de estatura, con 2 síntomas de depresión. 
Tabla 2.

Clúster identificados por Minera de datos.

\begin{tabular}{|c|c|c|c|c|c|c|c|}
\hline Clúster & Total & 0 & 1 & 2 & 3 & 4 & 5 \\
\hline $\mathrm{N}$ datos & 432 & 99 & 55 & 76 & 57 & 105 & 40 \\
\hline Sexo & & M & $\mathrm{H}$ & M & M & $\mathrm{H}$ & M \\
\hline Edad (años) & 16.5 & 16.6 & 17.6 & 16.8 & 16.9 & 16.2 & 15.5 \\
\hline Peso (Kg) & 57.0 & 51.1 & 68.8 & 59.8 & 72.2 & 54.0 & 51.6 \\
\hline Talla (cm) & 163 & 163.0 & 169.4 & 159.9 & 162.6 & 167.7 & 158.0 \\
\hline Índice de masa corporal kg/m² & 21.1 & 19.3 & 23.1 & 23.1 & 26.4 & 19.1 & 20.8 \\
\hline Perímetro cadera (cm) & 91.7 & 88 & 98.8 & 96.3 & 102.9 & 85.7 & 90.1 \\
\hline Perímetro cintura (cm) & 74.5 & 69.5 & 77.8 & 75.9 & 89.2 & 70.9 & 79.6 \\
\hline Pliegue bicipital (cm) & 26 & 24.8 & 28.2 & 27 & 30.5 & 24.2 & 24.9 \\
\hline Pliegue tricipital (cm) & 7.6 & 6.2 & 8.0 & 11 & 12.7 & 5.0 & 10.1 \\
\hline Pliegue sub escapular(cm) & 15.5 & 12.5 & 17.5 & 21 & 23 & 9.5 & 17.3 \\
\hline Pliegue supra iliaco (cm) & 12.7 & 10 & 14.5 & 16 & 23.5 & 9.5 & 16.1 \\
\hline Pliegue abdominal (cm) & 21.5 & 16.5 & 25.5 & 26 & 34.5 & 11.6 & 24.7 \\
\hline Índice cintura / cadera & .82 & .79 & .80 & .80 & .87 & .83 & .88 \\
\hline Perímetro cintura alterado & No & No & No & No & No & No & No \\
\hline Índice cintura / cadera alterado & No & No & No & No & $\mathrm{Si}$ & No & $\mathrm{Si}$ \\
\hline Índice de masa corporal alterado & No & No & No & No & $\mathrm{Si}$ & No & No \\
\hline Porcentaje de grasa (Slaughter) & 25.3 & 22.0 & 27.8 & 29.5 & 31.3 & 17.3 & 28.1 \\
\hline Porcentaje de grasa (Slaughter) alterado & No & No & $\mathrm{Si}$ & $\mathrm{Si}$ & $\mathrm{Si}$ & No & $\mathrm{Si}$ \\
\hline Estar muy cansado para hacer cualquier cosa (últimos 12 meses) & $\mathrm{Si}$ & $\mathrm{Si}$ & No & $\mathrm{Si}$ & $\mathrm{Si}$ & $\mathrm{Si}$ & $\mathrm{Si}$ \\
\hline Presentar problemas para dormir (últimos 12 meses) & $\mathrm{Si}$ & $\mathrm{Si}$ & No & $\mathrm{Si}$ & $\mathrm{Si}$ & No & No \\
\hline Sentirse triste, infeliz, deprimido (últimos 12 meses) & $\mathrm{Si}$ & $\mathrm{Si}$ & No & $\mathrm{Si}$ & $\mathrm{Si}$ & No & No \\
\hline Sentirse nervioso (últimos 12 meses) & $\mathrm{Si}$ & $\mathrm{Si}$ & No & $\mathrm{Si}$ & $\mathrm{Si}$ & No & $\mathrm{Si}$ \\
\hline Sentirse inútil (últimos 12 meses) & No & $\mathrm{Si}$ & No & No & $\mathrm{Si}$ & No & No \\
\hline Cambios en el apetito (últimos 12 meses) & $\mathrm{Si}$ & $\mathrm{Si}$ & No & $\mathrm{Si}$ & $\mathrm{Si}$ & No & No \\
\hline Ha sentido que lo tratan con menos respeto que a otras personas. & $\mathrm{Si}$ & $\mathrm{Si}$ & No & $\mathrm{Si}$ & $\mathrm{Si}$ & No & No \\
\hline Sentir que las personas actúan mejor que uno mismo (últimos 12 meses) & $\mathrm{Si}$ & $\mathrm{Si}$ & No & $\mathrm{Si}$ & $\mathrm{Si}$ & $\mathrm{Si}$ & No \\
\hline Le han puesto apodos o lo han insultado & No & No & No & No & $\mathrm{Si}$ & No & No \\
\hline Las personas se burlan del aspecto físico & No & No & No & No & $\mathrm{Si}$ & No & No \\
\hline Las personas se burlan del peso & No & No & No & No & Si & No & No \\
\hline
\end{tabular}

Nota. M (mujer); H (hombre) 


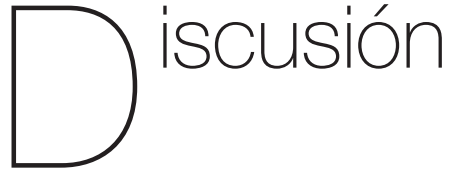

En adolescentes, los estudios que abordan la asociación entre obesidad y síntomas depresivos han venido en auge de la mano con el aumento de la prevalencia de ambas condiciones en este grupo etario. Luppino et al. (2010) presentan una revisión sistemática de esta asociación (obesidad vs. síntomas depresivos), encontrando que la relación entre estas variables es bidireccional, en decir, que la obesidad aumenta el riesgo de sufrir depresión y el diagnóstico de depresión es un predictor de la obesidad.

En esta misma línea, Quek et al. (2017) encontraron que existe una asociación positiva entre la obesidad en la infancia y la adolescencia y la depresión, de modo que los adolescentes y niños con esta condición tenían más síntomas depresivos que los individuos sanos o con sobrepeso. En el caso de este último grupo, los investigadores no encontraron una asociación significativa con la depresión.

Por otra parte, Mühlig, Antel, Föcker y Hebebrand (2016) hallaron que, si bien los estudios refieren una asociación entre la obesidad y la depresión, es difícil derivar conclusiones debido a la variedad metodológica de éstos, aunque sí observaron que la relación bidireccional entre estas variables parece ser más fuerte cuando se estudian en función del sexo, siendo mayor su poder predictivo en los grupos de niñas y mujeres adolescentes. Este último hallazgo coincide con algunos resultados de la presente investigación, pues se pudo observar que sólo en el grupo de mujeres adolescentes se presenta una relación significativa entre la presencia de síntomas depresivos y síntomas de obesidad, situación que no ocurre en el grupo de hombres, en donde, a pesar de observarse algunos síntomas depresivos, éstos no se relacionan con indicadores de obesidad.

Con relación a lo anterior, aunque en este estudio se halló una relación significativa entre los síntomas depresivos y los indicadores que determinan la obesidad, ésta se caracterizó por identificar aquellos síntomas depresivos que tenían un patrón similar (repetían) en los marcadores que determinan la obesidad. No se encontraron estudios similares al presente trabajo, por tanto, no fue posible comparar la naturaleza del patrón entre las variables consideradas en los clúster encontrados, de modo los resultados de este estudio deben ser considerados con precaución. Se requieren otros estudios y análisis que permitan obtener un mayor grado de comprensión de los patrones de las variables consideradas en este estudio. No obstante, se hallaron algunas publicaciones que han aplicado las técnicas de minería de datos a temas como la obesidad, realizando predicciones entre personas de mediana edad (Hossain, Mahmud, Hossin, Noori \& Jahan, 2018) o aplicadas a factores de riesgo (Ahamad, Ahmed, \& Uddin, 2016) y en depresión (Yoon, Taha, \& Bakken, 2014), de manera independiente.

Una de las limitaciones que tuvo este estudio fue la no utilización de una medida estandarizada para los síntomas depresivos, de modo que es difícil establecer un punto de referencia para 
la valoración clínica de los indicadores evaluados. Sin embargo, el cuestionario diseñado para tal fin tuvo en cuenta las características semiológicas de los trastornos depresivos señaladas por la Clasificación Internacional de Enfermedades CIE-10.

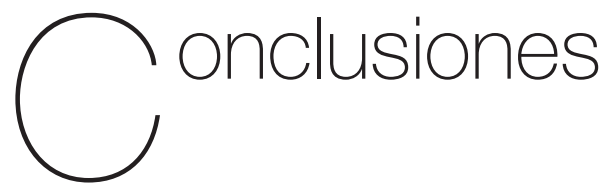

Las mujeres con mayor peso (clasificadas en sobrepeso) y con mayores índices antropométricos tienen todos los factores de depresión evaluados.

En la población estudiada se observó que, en las mujeres, a mayor peso presentan más factores de depresión.

Las mujeres con menor peso y menores índices antropométricos tienen hasta 3 factores de depresión no asociados a su peso.

Aunque un grupo de hombres sufre de factores de depresión, en ningún caso son asociados al peso de los participantes.

Las mujeres que sufren de todos los síntomas de depresión tienen estos factores: perímetro cintura/cadera, índice de masa corporal y porcentaje de grasa (Slaughter) elevados, comparado con el resto de la población.

Conflicto de intereses: Los autores declaran que no poseen conflicto de intereses.

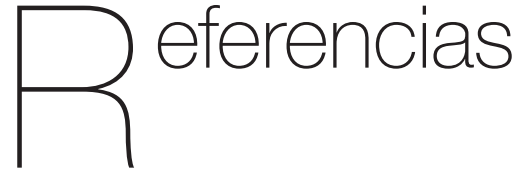

Ahamad, M.G., Ahmed, M.F., \& Uddin, M.Y. (2016). Clustering as data mining technique in risk factors analysis of diabetes, hypertension, and obesity. EJERS, European Journal of Engineering Research and Science, 1(6), 88-93. Recuperado de https://www.ejers.org/index.php/ejers/ article/view/202/110

Cabas, K., González, Y., \& Mendoza, C. (2018). Funcionamiento ejecutivo y depresión en universitarios con normopeso, sobrepeso y obesidad Tipo I. Informes Psicológicos, 18(1), 133-144. http://dx.doi.org/10.18566/ infpsic.v18n1a07

Curilem-Gatica, C., Rodríguez-Rodríguez, F., Almagià-Flores, A., Yuing-Farías, T., \& Berral F. (2016). Ecuaciones para la evaluación de la composición corporal en niños y adolescentes. Cadernos Saúde Pública, 32(7), 1-6. http://dx.doi. org/10.1590/0102-311X00195314.

De Onis, M., Onyango, A.W., Borghi, E., Siyam, A., Nishida, C., \& Siekmann, J. (2007). Development of a Who growth reference for school-aged children and adolescents. Bull World Health Organization, 85(9), 660-667.

Dipnall, J. F., Pasco, J. A., Berk, M., Williams, L. J., Dodd, S....\& Jacka, F. N. (2016). Fusing data mining, machine learning and traditional statistics to detect biomarkers associated with depression. PLoS One, 11(2), 1-23.

Fayyad, U., Piatetsky-Shapiro, G., \& Smyth, P. (1996). From data mining to knowledge discovery in databases. American 
Association for Artificial Intelligence, 17(3), 37-54.

Hossain, R., Mahmud, H., Hossin, A., Noori, S., \& Jahan, H. (2018). PRMT: Predicting Risk Factor of Obesity among Middle-Aged People Using Data Mining Techniques. Procedia Computer Science, 132, 1068-1076. https://doi.org/10.1016/j. procs.2018.05.022

Lasserre, A. M., Glaus, J., Vandeleur, C. L., Marques-Vidal, P., Vaucher, J., Bastardot, F., Waeber, G., Vollenweider, P., \& Preisig, M. (2014). Depression with atypical features and increase in obesity, body mass index, waist circumference, and fat mass: a prospective, population-based study. JAMA Psychiatry, 71(8), 880-888. https:// doi.org/10.1001/jamapsychiatry.2014.411

Lohman, T.G., Roche, A.F., \& Martorell, R. (Eds.). (1988). Anthropometric standardization reference manual. Champaign. IL: Human Kinetics

Luppino, F.S., De Wit, L.M., Bouvy, P.F., Stijnen, T., Cuijpers, P., Penninx, B.W., \& Zitman, F.G. (2010). Overweight, obesity, and depression: a systematic review and metaanalysis of longitudinal studies. Archives of General Psychiatry, 67(3), 220- 229.

Martinson, M. L., \& Vasunilashorn, S. M. (2016). The long-arm of adolescent weight status on later life depressive symptoms. Age and Ageing, 45(3), 389-395. https://doi. org/10.1093/ageing/afw020

Milano, W., Ambrosio, P., Carizzone, F., De Biasio, V., Di Munzio, W., Foia, M. G., \& Capasso, A. (2020). Depression and obesity: analysis of common biomarkers. Diseases (Basel, Switzerland), 8(2), 23-52. https://doi.org/10.3390/diseases8020023
Milovic, B. \& Milovic, M. (2012). Prediction and decision making in health care using data mining. International Journal of Public Health Science, 1(2), 69-78.

Ministerio de Salud (2010). Presentación de lanzamiento de Encuesta Nacional de la Situación Nutricional ENSIN 2010. Recuperado de https://www.icbf.gov.co/ sites/default/files/resumenfi.pdf

Ministerio de Salud (2015). Presentación de lanzamiento de Encuesta Nacional de la Situación Nutricional ENSIN 2015. Recuperado de https://www.minsalud.gov. co/Paginas/Gobierno-presenta-EncuestaNacional-de-Situaci\%C3\%B3n-Nutricionalde-Colombia-ENSIN-2015.aspx

Ministerio de Salud. (2017). Boletín de salud mental: Depresión. Subdirección de Enfermedades No Transmisibles. Recuperado de https://www.minsalud.gov. co/sites/rid/Lists/BibliotecaDigital/RIDE/ VS/PP/ENT/boletin-depresion-marzo-2017. pdf

Mühlig, Y., Antel, J., Föcker, M., \& Hebebrand, J. (2015). Are bidirectional associations of obesity and depression already apparent in childhood and adolescence as based on high-quality studies? A systematic review. Obesity Reviews, 17(3), 235-249. https://doi. org/10.1111/obr.12357

Ni, H., Yang, X., Fang, C., Guo, Y., Xu, M. \& He, Y. (2014). Data mining-based study on submentally healthy state among residents in eight provinces and cities in China. Journal of Traditional Chinese Medicine, 34(4), 511-517.

Organización Mundial de la Salud OMS. (1994). Guía de bolsillo de la clasificación de la CIE10. Madrid: Panamericana. 
Organización Mundial de la Salud OMS. (2017). Depresión. Recuperado de http://www. who.int/topics/depression/es/

Organización Mundial de la Salud OMS. (2015). Enfermedades Cardiovasculares. Recuperado de http://www.who.int/ mediacentre/factsheets/fs317/es/

Organización Mundial de la Salud OMS. (2002). Temas de salud: Definición y evaluación de riesgos en salud. Recuperado de https:// www.who.int swhr

Pereira-Miranda, E., Costa, P., Queiroz, V., Pereira-Santos, M., \& Santana, M. (2017). Overweight and obesity associated with higher depression prevalence in adults: a systematic review and metaanalysis. Journal of the American College of Nutrition, 36(3), 223-233. doi: 10.1080/07315724.2016.1261053.

Pirooznia, M., Seifuddin, F., Judy, J., Mahon, P., Potash, J., \& Zandi, P. (2012). Data mining approaches for genome-wide asscociation of mood disorders. Psychiatric Genetics, 22(2), 55-61.

Quek, Y., Tam, W., Zhang, M. \& Ho, R. (2017). Exploring the association between childhood and adolescent obesity and depression: a meta-analysis. Obesity Reviews, 18(7), 742-754. https://doi. org/10.1111/obr.12535

Ranta, K., Väänänen, J., Fröjd, S., Isomaa, R., Kaltiala-Heino, R., \& Marttunen, M. (2017). Social phobia, depression and eating disorders during middle adolescence: longitudinal associations and treatment seeking. Nordic Journal of Psychiatry, 71(8), 605-613. doi: 10.1080/08039488.2017.1366548

Weka Company (s.f.). Software Weka.Waikato Environment for Knowledge Analysis. Versión 3.8. Recuperado de https://www. cs.waikato.ac.nz/ml/weka/

Stice, E., \& Desjardins, C. (2018). Interactions between risk factors in the prediction of onset of eating disorders: Exploratory hypothesis generating analyses. Behaviour Research and Therapy, 105, 52-62. https:// doi.org/10.1016/j.brat.2018.03.005

Two Crows Corporation. (2005). Introduction to Data Mining and Knowledge Discovery. Recuperado de http://www.stat.ucla. edu/ hqxu/stat19/intro-dm.pdf

Villa-Roel, C., Buitrago, A., Rodríguez, D., Cano, D., Martínez, M.P., Camacho, P.A, Ruiz, A., \& Durán, A. (2009). Prevalence of metabolic syndrome in scholars from Bucaramanga, Colombia: a populationbased study. Study protocol. BMC Pediatrics, 9(28), 1-6. Recuperado de https://bmcpediatr.biomedcentral.com/ articles/10.1186/1471-2431-9-28

Yoon, S., Taha, B., \& Bakken S. (2014). Using a data mining approach to discover behavior correlates of chronic disease: a case study of depression. Studies in Health Technology and Informatics, 201, 71-78. Recuperado de https://www.ncbi.nlm.nih.gov/pmc/articles/ PMC4580372 


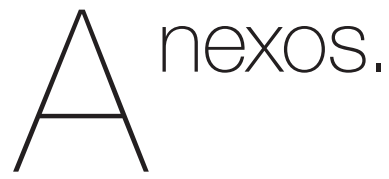

Material suplementario. Ecuación de Slaughter para determinar el Porcentaje de Grasa Corporal (\% GC)

\begin{tabular}{lc}
\hline \multicolumn{1}{c}{ Población } \\
\hline Mujeres \\
\hline Pre-púberes y púberes & $\% G C=1.33$ (P. tricipital + P. subescapular) $-.013\left(\right.$ P. tricipital + P. subescapular) ${ }^{2}-2.5$ \\
\hline Hombres & $\% G C=1.21$ (P. tricipital + P. subescapular) $-.008\left(\right.$ P. tricipital + P. subescapular ${ }^{2}-1.7$ \\
\hline Pre-púberes & $\% G C=1.21$ (P. tricipital + P. subescapular) $-.008\left(\right.$ P. tricipital + P. subescapular) ${ }^{2}-3.4$ \\
Púberes &
\end{tabular}

Nota. P. (Pliegue) 